\title{
Estudio comparativo sobre ansiedad matemática entre estudiantes de Costa Rica y España ${ }^{1}$
}

\author{
Comparative Study on Mathematical Anxiety Among Students From Costa Rica and Spain ${ }^{2}$
}

Estudo comparativo sobre ansiedade matemática entre estudantes da Costa Rica e Espanha ${ }^{3}$

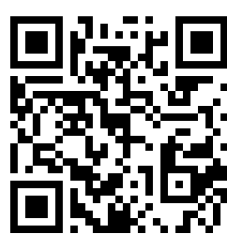

Islande Delgado-Monge

Universidad Estatal a Distancia

San José, Costa Rica

idelgado@uned.ac.cr

https://orcid.org/0000-0001-8344-7323

Enrique Castro-Martínez

Universidad de Granada

Granada, España

ecastro@ugr.es

https://orcid.org/0000-0002-2786-500X

Patricia Pérez-Tyteca

Universidad de Alicante

Alicante, España

patricia.perez@ua.es

https://orcid.org/0000-0002-7796-9042

Recibido • Received • Recebido: 17 / 10 / 2018

Corregido • Revised • Revisado: 20 / 02 / 2020

Aceptado • Accepted $\cdot$ Aprovado: 30 / 03 / 2020

Resumen: La ansiedad matemática (AM) es una de las reacciones afectivas que mayor influencia presenta en el desempeño del estudiantado; factores como el rendimiento académico, el género y la escogencia de la titulación se ven negativamente afectados por este constructo afectivo. En estudios anteriores se concluyó que tanto en Costa Rica como en España se han encontrado estudiantes que

\footnotetext{
${ }^{1}$ El manuscrito se deriva de un Trabajo Final de Graduación titulado: Estudio comparativo sobre ansiedad matemática entre estudiantes de Costa Rica y España.

${ }^{2}$ This paper is derived from a Graduation work "Estudio comparativo sobre ansiedad matemática entre estudiantes de Costa Rica y España".

${ }^{3}$ O manuscrito é derivado de um trabalho de graduação final intitulado "Estudio comparativo sobre ansiedad matemática entre estudiantes de Costa Rica y España".
} 
http://doi.org/10.15359/ree.24-2.15

ROR: https://ror.org/01t466c14 Universidad Nacional, Costa Rica

http://www.una.ac.cr/educare

educare@una.cr

presentan ansiedad matemática, por lo tanto, en este artículo científico derivado de una investigación se profundizó en ello y se propuso como objetivo realizar un estudio comparativo sobre AM entre el estudiantado de la Universidad Nacional Costa Rica y de la Universidad de Granada en España, y precisar esta comparación observando lo que ocurre en cada país respecto a las diferencias de género y a la escogencia de la titulación. Para ello se aplicó una escala tipo Likert a un grupo de 2085 estudiantes que cursaban la primera materia de matemática de su carrera universitaria, de este, 895 son estudiantes de la Universidad Nacional de Costa Rica y 1190 son estudiantes de la Universidad de Granada en España. La escala permitió agrupar los ítems en tres categorías: ansiedad matemática ante los problemas, ansiedad matemática ante los exámenes, y ansiedad ante la matemática en general; en esta última categoría se incluyen aquellas situaciones relacionadas con el transcurso habitual de las clases de matemática. La investigación es de tipo cuantitativa. Para el análisis de utilizó un diseño factorial en el que las medidas de la ansiedad matemática y la de sus subconstructos actúan como variables dependientes, y la nacionalidad del estudiantado, el sexo y el tipo de carrera que cursan son los factores considerados en dicho diseño. Se evidenció que el alumnado de ambos países mostró un comportamiento similar con respecto a su ansiedad matemática; además, tanto en Costa Rica como en España, el estudiantado de las carreras con la mayor cantidad de cursos de matemática presenta ansiedad matemática, ansiedad matemática ante los problemas, ansiedad matemática ante los exámenes y ansiedad ante la matemática en general, significativamente menor que el resto del alumnado. No encontramos diferencias estadísticamente significativas del país según el género, evidenciando que en ambos países se mantiene que las mujeres presentan mayor ansiedad matemática, ansiedad matemática ante los problemas, ansiedad matemática ante los exámenes y ansiedad ante la matemática en general que los hombres. También, encontramos de forma significativa una correlación positiva entre las variables dependientes.

Palabras claves: Ansiedad matemática; estudio comparativo; país; género; titulación; estudiantes universitarios.

Abstract: Mathematical anxiety is one of the affective reactions that have the most significant influence on students' performance. This affective construct negatively influences factors such as academic performance, gender, and the choice of the degree. Earlier studies concluded that there are students in Costa Rica and Spain who suffer from mathematical anxiety; so this article, derived from a research project, deeply analyzes the phenomenon and compares its presence in university students from the National University of Costa Rica and the University of Granada in Spain, and specifies this comparison observing what happens in each country in relation to gender differences and the choice of the degree program. A Likert scale was used to collect the information, which even allowed grouping the data into three categories: mathematical anxiety when facing problems, mathematical anxiety at the moment of exams, and mathematical anxiety in general. In this last category are included situations while teaching mathematics classes. The research is quantitative. The analysis implemented a factorial design in which the measures of mathematical anxiety and its sub-constructs act as dependent variables, and the nationality of the students, sex, and type of career students are enrolled in are the factors considered in the said design. The research revealed that the students of both countries showed similar behaviors concerning their mathematical anxiety. Besides, both in Costa Rica and in Spain, the students with a greater quantity of mathematics courses showed mathematical anxiety, mathematical anxiety when facing problems, mathematical anxiety at the moment of exams, and mathematical anxiety in general, significantly less than the rest of 
students. We did not find statistically significant differences based on gender in the country; this evidenced that, in both countries, it is accepted that women show higher mathematical anxiety when facing problems, mathematical anxiety at the moment of exams, and mathematical anxiety in general than men. We also found a significant positive correlation between the dependent variables.

Keywords: Mathematical anxiety; comparative study; country; gender; degree; university students.

Resumo: $A$ ansiedade matemática ( $A M)$ é uma das reações afetivas que mais influenciam no desempenho dos estudantes. Fatores como desempenho acadêmico, gênero e a escolha do grau são influenciados negativamente por esse construto afetivo. Em estudos anteriores concluiu-se que tanto Costa Rica e Espanha encontraram estudantes que têm ansiedade matemática, por isso, nesse artigo científico proveniente de uma pesquisa foi realizar um estudo comparativo sobre AM entre estudantes da Universidade Nacional da Costa Rica e da Universidade de Granada, na Espanha e especificar na comparação o que acontece em cada país em relação a diferenças de gênero e escolha de grau. Para lograr este objetivo, foi aplicada uma escala de Likert a 2085 estudantes da primeira matéria de matemática de sua carreira universitária, dos quais 895 são estudantes da Universidade Nacional da Costa Rica e 1190 são estudantes da Universidade de Granada, na Espanha. A escala permitiu agrupar os itens em três categorias: ansiedade matemática frente aos problemas, ansiedade matemática frente aos exames e ansiedade matemática em geral; nesta última categoria estão incluídas as situações relacionadas ao curso normal das aulas de matemática. A pesquisa é quantitativa. Para a análise utilizou um desenho fatorial no qual a ansiedade matemática e suas sub-construções agem como variáveis dependentes, e a nacionalidade dos estudantes, o sexo e tipo de carreira que concorrem são os fatores considerados neste projeto. Evidenciou-se que os estudantes dos dois países apresentaram um comportamento semelhante em relação à sua ansiedade matemática. Além disso, tanto Costa Rica como na Espanha, os estudantes com o maior número de cursos de matemática apresentam ansiedade matemática, ansiedade matemática frente aos problemas, ansiedade matemática frente aos exames e ansiedade matemática em geral significativamente inferior ao restantes dos estudantes. Não houve diferenças estatisticamente significativas relacionadas ao gênero, demonstrando que ambos países mantiveram as mulheres com maior ansiedade matemática, ansiedade matemática frente aos problemas, ansiedade matemática frente aos exames e ansiedade matemática em geral que os homens. Além disso, encontramos uma correlação positiva significativa entre as variáveis dependentes.

Palavras-chaves: Ansiedade matemática; estudo comparativo; país, gênero; grau; estudantes universitários.

\section{Introducción}

La ansiedad hacia las matemáticas presenta una función primordial dentro de los factores afectivos. Por esta razón, estudiarla se convierte en un aspecto sumamente necesario para el proceso de enseñanza-aprendizaje de las matemáticas. Tal y como indica Marshall (2000), es una importante pero poco entendida realidad para el estudiantado y un aspecto dirigido de manera descuidada por una parte de docentes. 
http://doi.org/10.15359/ree.24-2.15

ROR: https://ror.org/01t466c14 Universidad Nacional, Costa Rica

http://www.una.ac.cr/educare

educare@una.cr

Existen muchos factores que afectan al rendimiento del estudiantado en matemáticas. La ansiedad matemática es uno de ellos y sus efectos alcanzan a estudiantado de distintos niveles escolares, incluido el universitario (Pérez-Tyteca et ál., 2011). La ansiedad matemática no se manifiesta en todo el estudiantado por igual y tiene repercusión en la elección de su carrera universitaria y, por ende, en su futura profesión (Pérez-Tyteca, 2012). Estudios indican que estudiantes con mayor motivación y agrado hacia las matemáticas, alcanzan mayor nivel de desempeño en la materia (Petriz et ál., 2010).

El conocimiento de la ansiedad del estudiantado es un paso previo y fundamental para diseñar protocolos con el fin de mejorar el proceso de enseñanza-aprendizaje, por ello es una temática vigente y primordial. Países con buen rendimiento académico en matemáticas promulgan una experiencia positiva con respecto a los trabajos realizados en ansiedad matemática y agregan que es sumamente posible trabajar la temática con gran éxito (Organización para la Cooperación y el Desarrollo Económico [OCDE], 2004). Por este motivo, consideramos esencial realizar estudios que profundicen en la problemática existente en torno a la ansiedad matemática que siente el estudiantado. Con este fin hemos diseñado el presente estudio, que tiene como objetivo profundizar en la naturaleza de las reacciones ante la ansiedad matemática del estudiantado universitario novel de España y Costa Rica.

Estos son dos países que desarrollan, de manera paralela, sus planes de estudio. En ambos se trabajan los siguientes bloques: (a) números; (b) geometría; (b) medidas; (c) relaciones y álgebra; y (d) estadística y probabilidad, de manera que en cada curso se va profundizando cada vez más en los temas. Además, tanto en España como en Costa Rica, dentro de cada uno de los bloques se le da especial importancia a la resolución de problemas, aspecto que también abordamos en esta investigación.

Al realizar una comparación entre los resultados obtenidos en dos países diferentes, se contribuye con información relevante adicional a la ya existente sobre el grado de ansiedad matemática del estudiantado de ambos países, aportando información tanto específica de cada país, como de la comparación entre ambos. Con ello se contribuye a la generalización de la información obtenida.

Esta comparativa ayuda a vislumbrar problemáticas comunes que permitan extrapolar medidas paliativas de un país a otro. Es decir, como en Costa Rica no se han llevado a cabo prácticas concretas para reducir la ansiedad y en España sí, analizamos si las características son comunes, es decir, si en Costa Rica existe la misma problemática que en España, y que esto permita aplicar en Costa Rica los mismos programas de intervención que se han aplicado en España y que se ha visto que contribuyen a reducir la ansiedad matemática. 


\section{Antecedentes}

El vínculo entre ansiedad matemática y rendimiento es uno de los más abordados en las investigaciones que se han realizado (Palacios et ál., 2013). En ellas se ha hallado que una alta ansiedad matemática está significativamente relacionada con un bajo rendimiento académico (Pérez-Tyteca et ál., 2009).

Por otro lado, las diferencias de género en ansiedad matemática se han investigado a nivel internacional en todos los niveles educativos; en Costa Rica se ha iniciado su investigación en alumnado de secundaria y del nivel universitario. En muchos de esos trabajos se concluye que las mujeres presentan mayor ansiedad matemática que los hombres (Delgado, 2015; Fennema y Sherman, 1976; Pérez-Tyteca, 2012; Rodríguez et ál., 2012).

Aunque existen algunos resultados discordantes como son los de Perina (2002), quien debate la existencia de estas diferencias, indicando que pueden deberse a que las mujeres tienden a admitir sus sentimientos de ansiedad más que los hombres. De la Torre et ál. (2009) encuentran diferencias de género; pero en este caso las mujeres presentan menor ansiedad que los hombres. O Petriz et ál. (2010) afirman que una dosis de ansiedad matemática conlleva a un mayor desempeño en la materia.

También se ha investigado la relación entre la resolución de problemas y la ansiedad matemática. Por ejemplo, en muchas de las definiciones de ansiedad matemática, la resolución de problemas está presente. Asimismo, se evidencia la importancia del proceso de resolución de problemas en los instrumentos empleados para determinar la ansiedad matemática, ya que se hace alusión a este proceso en muchos de los ítems.

En Costa Rica se han realizado escasos trabajos sobre ansiedad matemática, mientras en España sí se han desarrollado diversos estudios. Los trabajos que se utilizarán para realizar este estudio comparativo son dos. El de Costa Rica, por Delgado (2015), quien estudió la ansiedad matemática en el estudiantado del curso Matemática General (MAT-001), su comportamiento y variables relacionadas a esta. Y el de España realizado por Pérez-Tyteca (2012) donde trabajó con 1242 estudiantes que iniciaban sus estudios en la Universidad de Granada, pertenecientes a 26 titulaciones diferentes, clasificadas en 5 niveles según su orientación científico-matemática. Pérez-Tyteca (2012) propone un modelo en donde la ansiedad matemática se ve influenciada de manera significativa por la ansiedad hacia los exámenes, la ansiedad hacia la resolución de problemas y la ansiedad hacia las matemáticas consideradas globalmente.

Los estudios comparativos sobre ansiedad matemática son escasos; a continuación, se comentarán los pocos trabajos encontrados. Ho et ál. (2000) realizaron un estudio sobre las dimensiones afectiva y cognitiva de la ansiedad matemática. Analizaron una muestra de estudiantes de sexto grado de China, Taiwán y Estados Unidos. Confirmaron en los tres países, 
http://doi.org/10.15359/ree.24-2.15

ROR: https://ror.org/01t466c14 Universidad Nacional, Costa Rica

http://www.una.ac.cr/educare

educare@una.cr

una distinción entre ambas dimensiones y una correlación negativa y significativa entre la dimensión afectiva de la ansiedad matemática y el rendimiento académico en la materia.

Por su parte, Lee (2009) investiga la relación entre los constructos autoconcepto en matemática, autoeficacia en matemática y ansiedad matemática. Para ello utiliza los datos de las pruebas PISA aplicadas a 41 países en 2003. Encuentra, entre otros datos, que países como Corea y Japón presentan alta ansiedad matemática y alto rendimiento en la materia. Pero países europeos como Finlandia, Holanda, Liechtenstein y Suiza, manifiestan baja ansiedad matemática y alto rendimiento académico.

Mohamed y Tarmizi (2010) realizaron un estudio entre estudiantes de secundaria de Tanzania y de Malasia. Compararon la ansiedad matemática del estudiantado de ambos países y su relación con el rendimiento académico. Encontraron mayor ansiedad matemática en estudiantes de Malasia que en el estudiantado de Tanzania. No encontraron diferencias significativas entre el género y la ansiedad matemática del estudiantado de ambos países.

En relación con los subconstructos, Pérez-Tyteca (2012) encontró que estudiantes presentan una mayor ansiedad matemática ante los exámenes que ante los problemas y a su vez, esta ansiedad matemática ante los problemas es mayor que la ansiedad ante la matemática en general.

Por lo tanto, el objetivo de este trabajo fue realizar un estudio comparativo sobre la ansiedad matemática entre estudiantes de Costa Rica y España; puntualmente, se comparó la ansiedad matemática, la ansiedad matemática ante los problemas, la ansiedad matemática ante los exámenes y la ansiedad ante la matemática en general del estudiantado de la Universidad Nacional, Costa Rica y de la Universidad de Granada en España. Se analizó si estas variables estaban mediatizadas en cada país según género y la escogencia de la titulación. Por último, se estudió la asociación entre los subconstructos y la asociación entre la ansiedad matemática y cada uno de estos subconstructos.

\section{Marco teórico}

Los afectos (emociones, actitudes y creencias) del alumnado son factores fundamentales para la comprensión de su conducta en matemáticas (Gil et ál., 2005). Al respecto, Hidalgo et ál. (2013) indican que tanto el proceso de aprendizaje induce reacciones afectivas como el afecto condiciona el proceso de enseñanza-aprendizaje.

McLeod (1989) define el dominio afectivo como un extenso rango de sentimientos y humores (estados de ánimo), generalmente considerados como algo diferente de la pura cognición, e incluye como componentes específicos de este dominio las actitudes, creencias y emociones. 
http://doi.org/10.15359/ree.24-2.15

ROR: https://ror.org/01t466c14 Universidad Nacional, Costa Rica http://www.una.ac.cr/educare

educare@una.cr

Las actitudes presentan mayor intensidad que las creencias y menor estabilidad, poseen un componente cognitivo (influenciada por las creencias) y una afectiva (influenciada por las emociones). Y las emociones son componentes afectivos, que presentan gran intensidad; pero no estabilidad. Además, las creencias están basadas en la experiencia y las actitudes son predisposiciones.

\section{Ansiedad matemática}

Como ya se mencionó en la introducción, la ansiedad matemática es una de las reacciones afectivas que mayor influencia tiene en el desempeño del estudiantado. De acuerdo con Hannula (2011), aunque las emociones de un estudiante o una estudiante pueden fluctuar y cambiar rápidamente durante la resolución de un problema, el estudiantado también tiene patrones emocionales muy estables en el tiempo. Por este motivo, muchos instrumentos de medida hacen referencia a la ansiedad matemática del alumnado a lo largo de toda su carrera estudiantil, lo que dota a este constructo de características de la actitud. Esta es la perspectiva que se adopta en el presente trabajo.

Richardson y Suinn (1972) definen la ansiedad matemática como el sentimiento de tensión y ansiedad que interfieren en la manipulación de números y en la resolución de problemas matemáticos en una amplia variedad de situaciones tanto cotidianas como académicas.

Así mismo, Tobias y Weissbroad (1980) manifiestan que la ansiedad matemática describe el pánico, indefensión, parálisis y desorganización mental que surgen cuando a un sujeto se le exige resolver un problema matemático. Fennema y Sherman (1976) sugieren que la ansiedad matemática consiste en una serie de sentimientos de ansiedad, terror, nerviosismo y síntomas físicos asociados que surgen al hacer matemáticas (p. 4).

Pérez-Tyteca (2012) concluye que la ansiedad matemática es:

Un estado afectivo caracterizado por la ausencia de confort que puede experimentar un individuo en situaciones relacionadas con las matemáticas, tanto de su vida cotidiana como académica, y que se manifiesta mediante un sistema de respuestas que engloban una serie de "síntomas", como son: tensión, nervios, preocupación, inquietud, irritabilidad, impaciencia, confusión, miedo y bloqueo mental. (p.20)

Dadas las consideraciones anteriores, la definición escogida en este trabajo de investigación es la de Pérez-Tyteca (2012), ya que acoge de forma muy coherente las definiciones anteriormente mencionadas. 
http://doi.org/10.15359/ree.24-2.15

ROR: https://ror.org/01t466c14 Universidad Nacional, Costa Rica

http://www.una.ac.cr/educare

educare@una.cr

\section{Subconstructos de ansiedad matemática}

La escala de ansiedad matemática utilizada en este trabajo permite agrupar los ítems en tres categorías: ansiedad matemática ante los problemas, ansiedad matemática ante los exámenes, y ansiedad ante la matemática en general; en esta última categoría se incluyen aquellas situaciones relacionadas con el transcurso habitual de las clases de matemática. Esta agrupación ya se ha utilizado en trabajos precedentes, como el de Pérez-Tyteca (2012) o el de Sánchez-Mendías (2013).

\section{Metodología}

Para estudiar las comparaciones del nivel de ansiedad según las variables consideradas hemos utilizado un diseño factorial en el que las medidas de la ansiedad matemática y la de sus subconstructos actúan como variables dependientes; la nacionalidad del estudiantado, el sexo y el nivel de orientación científico-matemática de la titulación (que se explicará en la sección de variables) son los factores considerados en dicho diseño. Esta investigación es de tipo cuantitativa.

\section{Propósito de la investigación}

El propósito de este trabajo fue realizar un estudio comparativo sobrela ansiedad matemática entre estudiantes de Costa Rica y España. Para ello se comparó el nivel de ansiedad matemática de estudiantes de la Universidad Nacional, Costa Rica y de la Universidad de Granada en España y se precisó esta comparación observando lo que ocurre en cada país respecto a las diferencias de género y al nivel de orientación científico-matemática de la titulación. La comparación de ambos colectivos también se realizó según los subconstructos de ansiedad definidos en Pérez-Tyteca (2012); concretamente, se comparó la ansiedad matemática ante los problemas, la ansiedad matemática ante los exámenes y la ansiedad ante la matemática en general del estudiantado de la Universidad Nacional, Costa Rica y de la Universidad de Granada en España y se analizó si estas variables estaban mediatizadas en cada país según género y nivel de orientación científicomatemática de la titulación. Por último, se estudió la asociación entre los subconstructos y la asociación entre la ansiedad matemática y cada uno de estos subconstructos.

\section{Muestra}

La muestra total de este trabajo está compuesta por 2085 estudiantes que cursaban la primera materia de matemática de su carrera universitaria. De ellos, 895 son estudiantes de la Universidad Nacional, Costa Rica y 1190 son estudiantes de la Universidad de Granada en España. En ambos colectivos participaron estudiantes de sexo masculino y femenino. De Costa 
Rica, 428 son hombres y 467 son mujeres, mientras que de la Universidad de Granada, 464 son hombres, 726 son mujeres.

El estudiantado costarricense pertenecía a las carreras de Ingeniería en Sistemas, Ingeniería Agrónoma, Biología, Economía, Teología, Enseñanza de la Matemática, Filosofía, Enseñanza de la Religión, Sociología, Planificación y Promoción Social, Administración de Empresas, Enseñanza de las Ciencias, Ingeniería en Ciencias Forestales, Ingeniería en Topografía y Catastro, Ingeniería en Gestión Ambiental, Comercio y Negocios Internacionales, Cartografía y Diseño Digital y Ciencias Geográficas.

El estudiantado español pertenecía a las titulaciones de Arquitectura, Arquitectura Técnica, Ciencias Empresariales, Enfermería, Estadística, Óptica, Ingeniería Telecomunicación, Ingeniería Informática, Ingeniería Química, Ingeniería de Gestión, Ingeniería en Sistemas, Administración y Dirección de Empresas, Biología, Relaciones Laborales, Economía, Farmacia, Física, Geología, Matemáticas, Química, Biblioteconomía y Documentación, Ciencias Políticas y Derecho, Sociología, Educación Infantil y Educación Primaria.

\section{Instrumento}

Para la recogida de datos se utilizó una traducción y adaptación -ya utilizada en trabajos previos como los de Pérez-Tyteca (2012) o Sánchez-Mendías (2013)- de la subescala de Fennema y Sherman (1976) que consta de 12 ítems. Es una escala tipo Likert con 5 opciones de respuesta enumeradas de la siguiente manera: 1, 2, 3, 4 y 5, que corresponden respectivamente a totalmente en desacuerdo, bastante en desacuerdo, ni de acuerdo ni en desacuerdo, bastante de acuerdo y totalmente de acuerdo.

Se utilizó la fiabilidad compuesta y la varianza media extractada para medir la fiabilidad de la escala, donde la fiabilidad compuesta debe ser superior a .7 y la varianza media extractada superior a .5. En el estudiantado de la Universidad Nacional, Costa Rica, estos valores fueron respectivamente 0.93 y .51; en el estudiantado de la Universidad de Granada en España estos valores fueron respectivamente .92 y .51 , lo cual evidencia gran fiabilidad del instrumento en ambos países.

\section{Procedimiento}

Tanto en Costa Rica como en España, los datos fueron recogidos a principio de curso, cuando el estudiantado todavía no estaba influenciado por la enseñanza universitaria. Las investigadoras supervisaron la aplicación de todos los cuestionarios; la profesora Patricia Pérez Tyteca en la Universidad de Granada en España y la profesora Islande Delgado Monge en la Universidad Nacional, Costa Rica. 
http://doi.org/10.15359/ree.24-2.15

ROR: https://ror.org/01t466c14 Universidad Nacional, Costa Rica

http://www.una.ac.cr/educare

educare@una.cr

\section{Variables}

Las variables dependientes consideradas en esta investigación son ansiedad matemática (AM) y los subconstructos de ansiedad matemática: (a) ansiedad matemática ante los problemas (AP); (b) ansiedad matemática ante los exámenes (AP); y (c) ansiedad ante la matemática en general (AG). Las variables independientes son la nacionalidad, el género y el nivel de orientación científico-matemático de la titulación (OCM).

La variable ansiedad matemática se obtuvo sumando las puntuaciones en cada ítem del instrumento y dividiendo este valor entre el total de ítems. Cuanto mayor sea el puntaje, mayor es la ansiedad matemática del estudiantado. La ansiedad matemática ante los problemas, la ansiedad matemática ante los exámenes y la ansiedad ante la matemática en general, también toman un valor continuo entre 1 y 5 . Cada una de estas variables dependientes se calcula con determinados ítems del instrumento aplicado.

La variable nacionalidad presenta dos valores: Costa Rica y España. La variable género toma también dos valores: hombre y mujer. Y la variable nivel de orientación científicomatemático de la titulación presenta cinco valores enumerados de 1 a 5, donde 1 corresponde a la orientación con menor enfoque científico-matemático y 5 la de mayor enfoque.

Con respecto a la variable nivel de orientación científico-matemática de la titulación, las carreras cursadas por los sujetos de la muestra se dividieron en 5 niveles de acuerdo con la cantidad de créditos de matemática del plan de estudios de la titulación. Los 5 niveles son: (a) N1; (b) N2; (c) N3; (d) N4; y (e) N5; donde un mayor nivel de orientación está asociado a una mayor cantidad de créditos de matemática de la carrera.

\section{Análisis de los datos}

Las comparaciones se organizaron en función de las variables independientes nacionalidad, género y OCM. Estas comparaciones se realizaron entre la muestra de estudiantes de la Universidad Nacional, Costa Rica y la muestra de la Universidad de Granada en España con respecto a la $A M$ y a los subconstructos $A P, A E$ y $A G$.

Para determinar si existen diferencias significativas entre los distintos niveles de las variables consideradas, se contrastó empíricamente empleando la técnica de modelo lineal general multivariante con un nivel de confianza de .05 por ciento; por ello, inicialmente se verificó el cumplimiento de los supuestos paramétricos. La independencia de los datos se cumple por ser poblaciones distintas; para la homocedasticidad se utilizó la prueba de Levene $p=.190$; y para el supuesto de normalidad construimos gráficos para evidenciar que la distribución presenta gran tendencia a una distribución normal. 
http://doi.org/10.15359/ree.24-2.15 ROR: https://ror.org/01t466c14 Universidad Nacional, Costa Rica http://www.una.ac.cr/educare educare@una.cr

\section{Resultados, análisis y discusión}

\section{Ansiedad matemática}

Sobre una puntuación máxima de cinco, en la Tabla 1 se recogieron las medias y las desviaciones típicas de los puntajes de ansiedad matemática otorgados por el estudiantado de la muestra. El puntaje promedio de ansiedad matemática presente en el estudiantado de la muestra de la Universidad Nacional, Costa Rica, fue de 2.78 y en la muestra de la Universidad de Granada en España fue de 2.73. Estos valores se obtuvieron sumando los datos correspondientes al puntaje promedio de ansiedad matemática de cada alumno y alumna, y dividiendo este valor entre el total de informantes.

Tabla 1: Estadísticos descriptivos de AM según País * Género * OCM

\begin{tabular}{|c|c|c|c|c|c|c|c|c|}
\hline \multicolumn{3}{|c|}{ País } & \multicolumn{5}{|c|}{ OCM } & \multirow[t]{2}{*}{ Total } \\
\hline & & & N1 & N2 & N3 & N4 & N5 & \\
\hline \multirow{3}{*}{ Costa Rica } & \multirow{2}{*}{ Género } & Hombre & $\begin{array}{c}2.48 \\
(1.46)\end{array}$ & $\begin{array}{l}2.79 \\
(.88)\end{array}$ & $\begin{array}{l}2.51 \\
(.79)\end{array}$ & $\begin{array}{l}2.69 \\
(.73)\end{array}$ & $\begin{array}{l}1.79 \\
(.25)\end{array}$ & $\begin{array}{l}2.61^{*} \\
(.79)^{* *}\end{array}$ \\
\hline & & Mujer & $\begin{array}{l}2.71 \\
(.43)\end{array}$ & $\begin{array}{l}3.09 \\
(.82)\end{array}$ & $\begin{array}{l}2.86 \\
(.85)\end{array}$ & $\begin{array}{l}3.05 \\
(.81)\end{array}$ & $\begin{array}{l}1.71 \\
(.87)\end{array}$ & $\begin{array}{l}2.94 \\
(.85)\end{array}$ \\
\hline & \multicolumn{2}{|l|}{ Total } & $\begin{array}{l}2.60 \\
(.97)\end{array}$ & $\begin{array}{l}2.97 \\
(.85)\end{array}$ & $\begin{array}{l}2.69 \\
(.84)\end{array}$ & $\begin{array}{l}2.85 \\
(.79)\end{array}$ & $\begin{array}{l}1.74 \\
(.72)\end{array}$ & $\begin{array}{l}2.78 \\
(.84)\end{array}$ \\
\hline \multirow{3}{*}{ España } & \multirow{2}{*}{ Género } & Hombre & $\begin{array}{l}2.84 \\
(.90)\end{array}$ & $\begin{array}{l}2.48 \\
(.73)\end{array}$ & $\begin{array}{l}2.53 \\
(.77)\end{array}$ & $\begin{array}{l}2.41 \\
(.71)\end{array}$ & $\begin{array}{l}2.20 \\
(.66)\end{array}$ & $\begin{array}{l}2.52 \\
(.78)\end{array}$ \\
\hline & & Mujer & $\begin{array}{l}2.97 \\
(.80)\end{array}$ & $\begin{array}{l}3.08 \\
(.79)\end{array}$ & $\begin{array}{l}2.70 \\
(.75)\end{array}$ & $\begin{array}{l}2.57 \\
(.63)\end{array}$ & $\begin{array}{l}2.53 \\
(.66)\end{array}$ & $\begin{array}{l}2.87 \\
(.78)\end{array}$ \\
\hline & \multicolumn{2}{|l|}{ Total } & $\begin{array}{l}2.94 \\
(.82)\end{array}$ & $\begin{array}{l}2.92 \\
(.82)\end{array}$ & $\begin{array}{l}2.63 \\
(.76)\end{array}$ & $\begin{array}{l}2.47 \\
(.68)\end{array}$ & $\begin{array}{l}2.34 \\
(.67)\end{array}$ & $\begin{array}{l}2.73 \\
(.80)\end{array}$ \\
\hline \multicolumn{3}{|c|}{ Total } & $\begin{array}{l}2.94 \\
(.82)\end{array}$ & $\begin{array}{l}2.95 \\
(.83)\end{array}$ & $\begin{array}{l}2.68 \\
(.82)\end{array}$ & $\begin{array}{l}2.65 \\
(.76)\end{array}$ & $\begin{array}{l}2.24 \\
(.71)\end{array}$ & $\begin{array}{l}2.75 \\
(.82)\end{array}$ \\
\hline
\end{tabular}

*Puntuación media.

**Desviación típica.

Nota: Elaboración propia.

Al aplicar la prueba ANOVA de contraste, las diferencias entre las medias de las puntuaciones del estudiantado de Costa Rica y España no resultaron estadísticamente significativas $F(1)=$ $.516 ; p=.472$. Esto indica que la ansiedad matemática encontrada en estudiantes de España y de Costa Rica presenta gran similitud (Tabla 2). 
http://doi.org/10.15359/ree.24-2.15

ROR: https://ror.org/01t466c14 Universidad Nacional, Costa Rica

http://www.una.ac.cr/educare

educare@una.cr

Tabla 2: Resultados del análisis de la varianza de AM según País * Género * OCM

(Diseño factorial $2 \times 2 \times 5$ )

\begin{tabular}{lcrccc}
\hline Variables & $\mathrm{df}$ & \multicolumn{1}{c}{$\mathrm{F}$} & $\mathrm{p}$ & Parcial ń $^{2}$ & Potencia observada \\
\hline País & 1 & .516 & .472 & .000 & .111 \\
Género & 1 & 7.919 & .005 & .004 & .803 \\
OCM & 4 & 8.028 & .000 & .015 & .998 \\
País * Género & 1 & .054 & .817 & .000 & .056 \\
País * OCM & 4 & 5.421 & .000 & .010 & .976 \\
Género * OCM & 4 & .859 & .488 & .002 & .276 \\
País * Género * OCM & 4 & 1.348 & .250 & .003 & .425 \\
Error & 2065 & & & & \\
\hline
\end{tabular}

$a=.05$

Nota: Elaboración propia.

Considerando el estudiantado de ambos países en conjunto, hemos encontrado efecto significativo de la variable Género $F(1)=7.919, p=.005$ (Tabla 2). La puntuación media de las mujeres $2.89(\sigma=.81)$ en ansiedad matemáticas es significativamente mayor que la de los hombres 2,56 $(\sigma=.79)$. Desde un punto de vista de la generabilidad de resultados, es importante analizar si estas diferencias de género se dan en los dos países.

Dado que la interacción entre género y país no resultó significativa $F(1)=.054 ; p=.817$, esto pone en evidencia que en ambos países se mantiene el comportamiento de que las mujeres presentan mayor ansiedad matemática que los hombres de manera significativa (Figura 1).

Figura 1: Ansiedad matemática por género y país

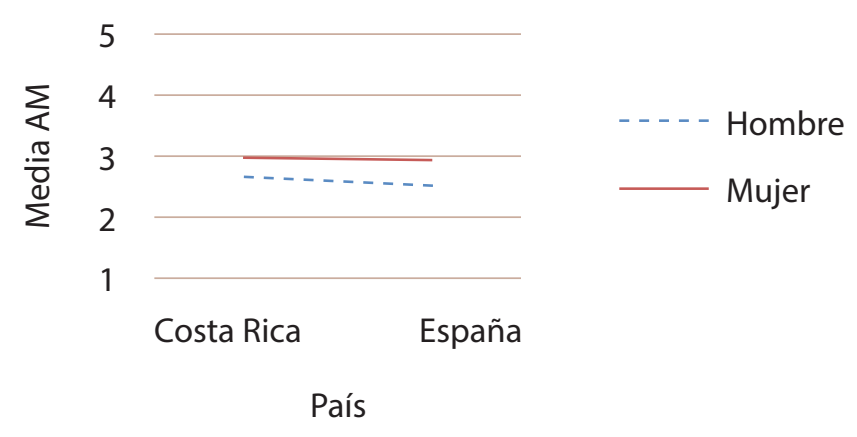

Nota: Elaboración propia. 
http://doi.org/10.15359/ree.24-2.15

ROR: https://ror.org/01t466c14 Universidad Nacional, Costa Rica

La variable OCM tiene efecto significativo sobre la puntuación de ansiedad matemática del estudiantado $F(4)=8.028, p=.000$. Puesto que esta variable tiene 5 niveles ello indica que hay diferencias significativas entre ellos.

Como la OCM está compuesta por 5 niveles, se aplicaron contrastes múltiples a posteriori mediante el método Scheffe ( $a=.05)$ para determinar entre cuáles niveles se daban diferencias significativas $y$, así, se encontraron tres agrupaciones significativamente homogéneas. El primer grupo está compuesto por el nivel 1 y el nivel 2; el segundo grupo por el nivel 3 y el nivel 4; y el tercer grupo por el nivel 5. También se observa que, con respecto a la OCM, el estudiantado de las titulaciones con mayor cantidad de cursos de matemática presentó menor AM (Tabla 1).

El efecto de la variable OCM no interactúa con el género de forma significativa $(F(4)=$ $.859, p=.488)$, pero sí tiene influencia en los resultados por países $(F(4)=5.421, p=.000)$. El efecto de esta interacción significativa se observa en la Figura 2.

Figura 2: Ansiedad matemática por OCM y país

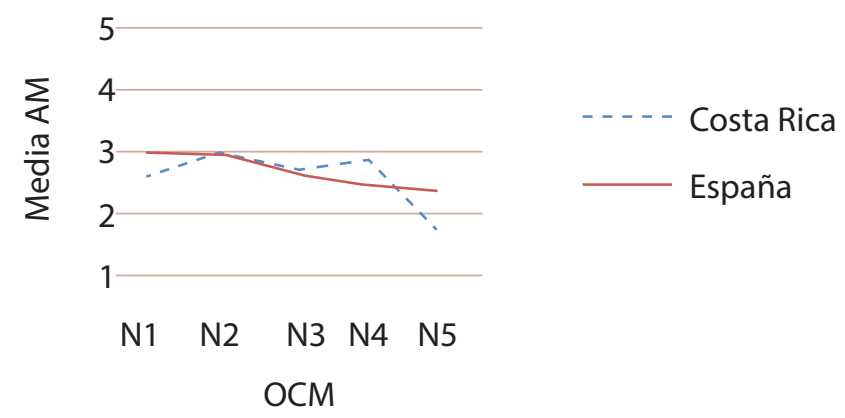

Nota: Elaboración propia.

Mientras en España la ansiedad matemática disminuye con respecto a la variable OCM desde el nivel 1 hasta el nivel 5 de forma lineal, en Costa Rica aumenta y disminuye de un nivel a otro. Cabe destacar el fuerte descenso del nivel de ansiedad del estudiantado de Costa Rica situados en el nivel 5 de OCM. El estudiantado de Costa Rica situado en los dos niveles extremos de la variable OCM tiene un nivel de ansiedad menor que el de España, por el contrario, los de los niveles medios están por encima.

Para la puntuación de ansiedad matemática no se ha obtenido efecto significativo sobre ella de las tres variables independientes OCM, género y país $F(4)=1.348 ; p=.250$. Por lo que el único efecto de interacción obtenido ha sido el de la variable OCM con el país.

\section{Correlación entre la AM y los subconstructos AP, AE y AG}

Los puntajes sobre AM obtenidos mediante la subescala de ansiedad de Fennema-Sherman pueden verse influenciados en mayor o menor medida por alguna de las componentes de esta 
http://doi.org/10.15359/ree.24-2.15

ROR: https://ror.org/01t466c14 Universidad Nacional, Costa Rica

http://www.una.ac.cr/educare

educare@una.cr

subescala: AP, AE y AG. Con el objeto de ver si los resultados obtenidos de manera general son más o menos consistentes con alguna de estos componentes o subconstructos, se ha estudiado si estos tres componentes se comportan de modo homogéneo. Para ello, se estudió la correlación entre los tres subconstructos AP, AE y AG, y la significatividad de estas correlaciones. El coeficiente de correlación de Pearson obtenido para cada una de las tres comparaciones posibles ha dado que las correlaciones entre AP y AE $r(2085)=.478 ; p<.001$, entre AP y AG $r(2085)=.694 ; p<.001$, y entre $A E$ y $A G r(2085)=.625 ; p<.001$ resultaron positivas y significativas.

Figura 3: Correlación de los subconstructos de ansiedad matemática

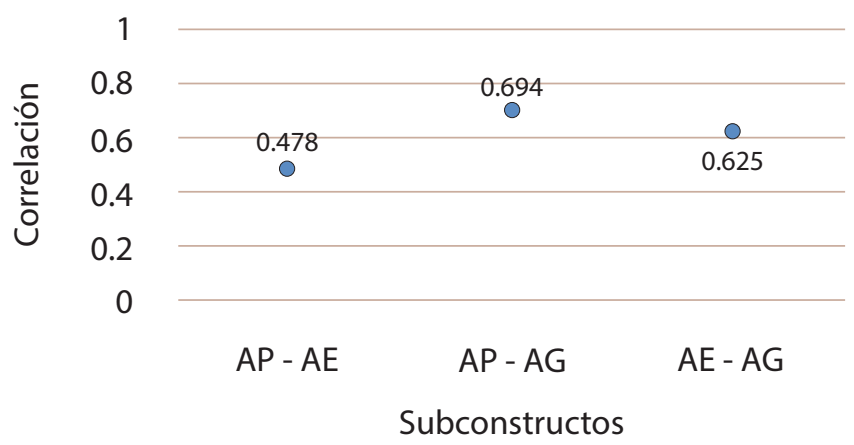

Nota: Elaboración propia.

Sin embargo, los valores de correlación obtenidos no son muy altos, por lo que se considera que los subconstructos afectan, de algún modo, los resultados generales obtenidos. Con el objeto de identificar cuál de las puntuaciones de las componentes concuerda más con la puntuación total en el test, se ha calculado la correlación entre la puntuación de cada subconstructo con la puntuación total.

Se encontró que la correlación entre la ansiedad matemática y AP $r(2085)=.817 ; p<.001$, entre la ansiedad matemática y AE $r(2085)=.763 ; p<.001$, y entre la ansiedad matemática y AG $r(2085)=.938 ; p<.001$, resultaron positivas y significativas (Figura 4).

Figura 4: Correlaciones entre la AM y los subconstructos AP, AE y AG.

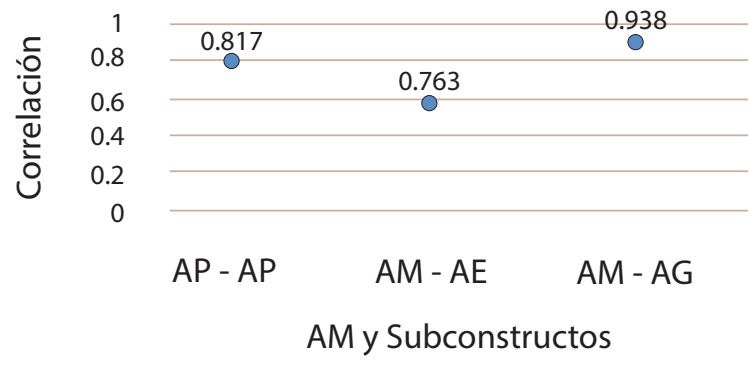

Nota: Elaboración propia. 
http://doi.org/10.15359/ree.24-2.15

ROR: https://ror.org/01t466c14 Universidad Nacional, Costa Rica

Estos valores de la correlación indican en cierto modo cuál de los subconstructos puntúa de forma más parecida en las respuestas de estudiantes en relación con la puntuación total en ansiedad matemática. Por ello, realizando el mismo análisis de la varianza factorial para cada subconstructo, se exponen los cambios que se han producido respecto a los resultados generales obtenidos.

\section{Análisis de los subconstructos de ansiedad matemática}

Para cada uno de los tres subconstructos considerados (AP, AE y AG) se han realizado análisis de la varianza factorial respecto a los factores país, género y OCM. La Tabla 3 recoge todos los efectos posibles de las variables estudiadas que han resultado significativos.

Tabla 3: Efectos significativos encontrados

\begin{tabular}{lcccc}
\multicolumn{1}{c}{ Variables } & AM & AP & AE & AG \\
\hline País & & $p<.05$ & $p<.05$ & \\
Género & $p<.05$ & $p<.05$ & $p<.05$ & \\
OCM & $p<.05$ & $p<.05$ & $p<.05$ & $p<.05$ \\
País * Género & & & & \\
País * OCM & $p<.05$ & $p<.05$ & & $p<.05$ \\
Género * OCM & & & & \\
País * Género * OCM & & & & \\
\hline
\end{tabular}

Nota: elaboración propia.

Con respecto al país, al aplicar la prueba ANOVA de contraste, las diferencias en el comportamiento de las medias de $\operatorname{AP} F(1)=6.880 ; p=.009$ y $\operatorname{AE} F(1)=7.739 ; p=.005$ entre ambos países resultaron estadísticamente significativas, no así para AG. La media de AP es mayor en España (2.70) que en Costa Rica (2.50) y la media de AE es mayor en Costa Rica (3.36) que en España (3.06). Así, se evidencia que solo existe similitud entre la puntuación media obtenida en $A M$ y en la del subconstructo $A G$, mientras que las puntuaciones medias de $A P$ y de $A E$ muestran un comportamiento diferente a AM.

La influencia del género ha resultado significativa tanto en AP como en $A E$, la media en ambos subconstructos es significativamente mayor en las mujeres que en los hombres, lo cual coincide con lo encontrado en AM. A diferencia de AG, donde no se encontró un efecto significativo con respecto al género $F(1)=2.215 ; p=.137$. Este resultado es importante en cuanto 
http://doi.org/10.15359/ree.24-2.15

ROR: https://ror.org/01t466c14 Universidad Nacional, Costa Rica

http://www.una.ac.cr/educare

educare@una.cr

pone en cuestión la afirmación general de que las mujeres siempre van a puntuar mayor que los hombres por su mayor grado de sinceridad.

El efecto significativo del tipo de carrera se mantiene en todas las variables. Al igual que $\mathrm{AM}$, los tres subconstructos presentaron un efecto significativo según OCM. Esto indica que existen diferencias entre los 5 niveles de los que consta esta variable. Por ello, se aplicaron los contrastes múltiples a posteriori mediante el método Scheffe $(\alpha=.05)$ para determinar entre cuáles niveles se presentaban estas diferencias.

Entre los niveles de AP se dan dos agrupaciones significativamente homogéneas. El primer grupo está compuesto por el nivel 1 y el nivel 2; y el segundo grupo por el nivel 3, el nivel 4 y el nivel 5. Entre los niveles de $A E$, existen dos grupos que son significativamente homogéneos. El primer grupo está compuesto por el nivel 1, el nivel 2, el nivel 3 y el nivel 4; y el segundo grupo por el nivel 5. Y entre los niveles de AG existen tres agrupaciones significativamente homogéneas. El primer grupo está compuesto por el nivel 1 y el nivel 2, el segundo grupo por el nivel 3 y el nivel 4, y el tercer grupo por el nivel 5.

El efecto de interacción según género y país no resultó significativo en las variables consideradas, es decir, el comportamiento en ambos países es muy similar en AM, AP, AE y AG. Por tanto, al analizar por separado los datos de los dos países, la puntuación dada en todas las variables es mayor en las mujeres que en los hombres, tanto en Costa Rica como en España

De igual forma que en AM, el efecto de la variable OCM en relación al país interactúa de forma significativa en AP y AG. Mientras en España la AP y la AG disminuye desde el nivel 1 hasta el nivel 5, en Costa Rica aumenta y disminuye de un nivel a otro. Surge una discordancia importante entre AE y AM cuando analizamos el comportamiento de los tipos de carrera según el país. El efecto de interacción entre AE y OCM deja de ser significativo.

Al igual que en $\mathrm{AM}$, tampoco se encontraron efectos significativos según la OCM y género, ni efectos de interacción triple país * Género * OCM.

\section{Conclusiones}

El objetivo de este trabajo fue realizar un estudio comparativo sobre la AM entre el estudiantado de Costa Rica y de España comparándola entre alumnado de la Universidad Nacional, Costa Rica, y de la Universidad de Granada en España. Precisamos esta comparación observando lo que ocurre en cada país respecto a las diferencias de género y a la OCM. Este proceso se reiteró de modo similar para los tres subconstructos de ansiedad matemática: AP, AE y AG.

La AP resultó significativamente mayor en el estudiantado español que en el costarricenses. Mientras AE resultó significativamente mayor en estudiantado costarricense que en español. No 
se localizaron estudios en los cuales se llevarán a cabo comparaciones de AP o AE entre países, lo cual refleja lo novedoso del trabajo que hemos realizado. Aunque podríamos relacionar estas diferencias con la manera de trabajar la resolución de problemas en ambos países, con el peso de los exámenes o la forma de evaluación, por ejemplo, podríamos incluso suponer que estas diferencias pueden ser causadas porque la resolución de problemas aplicada en España provoque mayor ansiedad al momento de trabajarla en el aula, pero mayor seguridad en el estudiantado al momento de realizar sus pruebas de evaluación sumativa como lo son los exámenes.

Con respecto al género en la muestra total, las mujeres presentaron una $A M, A P$ y $A E$ significativamente mayor que los hombres, resultado que concuerda con estudios como los de Delgado (2015), Pérez-Tyteca (2012), Pérez-Tyteca et ál. (2009), Fennema y Sherman (1976) y Valero (1999). Estas diferencias se producen pese al avance en cuestión de igualdad de género que hacen que hoy en día ya se hayan superado muchos de los prejuicios que sustentaban la idea de que la matemática era algo de hombres; de hecho, Perina (2002) debate la existencia de estas diferencias, indicando que pueden ser porque las mujeres tienden a mostrar más sinceridad que los hombres al expresar sus sentimientos. A este respecto, el hecho de que en este trabajo el género no presente un efecto significativo sobre la AG nos hace poner en cuestión esta afirmación.

Al estudiar la ansiedad matemática según género y país, no se encontraron efectos de interacción significativos entre estas dos variables, evidenciando que en ambos países se mantiene que las mujeres presentan de manera significativa una AM, AP y AE mayor que los hombres. Mohamed y Tarmizi (2010) realizaron un estudio similar, pero en su caso fueron los efectos de la ansiedad matemática en cuanto al género en cada país, los que no resultaron significativos. Se evidencia que la diferencia por género es una constante que se vive en ambos países, donde las mujeres se ven claramente afectadas en mayor medida, por ello se podrían realizar comparaciones entre más países y así determinar variables presentes en cada región que podrían estar influyendo.

En la OCM a nivel general, encontramos que el estudiantado de las carreras con la mayor cantidad de cursos de matemática, presentan una AM, AP, AE y AG significativamente menor que los restantes alumnados. Esta situación se relaciona con lo encontrado en el estudio realizado por Pérez-Tyteca (2012), donde estudiantes con mayor ansiedad matemática prefieren escoger titulaciones con la menor cantidad posible de cursos de matemática, implicando así una influencia negativa de la ansiedad matemática sobre la elección de la carrera universitaria.

Sí se ha encontrado, en este estudio, un efecto de interacción mutuo entre las variables OCM y país. Mientras en el estudiantado español la AM, AP, AE y AG va disminuyendo desde el nivel 1 hasta el nivel 5; en el estudiantado de Costa Rica aumenta y disminuye de un nivel a otro. 
http://doi.org/10.15359/ree.24-2.15

ROR: https://ror.org/01t466c14 Universidad Nacional, Costa Rica

http://www.una.ac.cr/educare

educare@una.cr

Es posible que estas diferencias puedan ser ocasionadas porque las titulaciones de Costa Rica y España presentan discrepancias entre sí, lo cual no permitió que la división entre los niveles fuese equivalente en los dos países.

No se han encontrado efectos de interacción mutua de las variables género y OCM con respecto a la ansiedad matemática, ni con respecto a cada uno de los tres subconstructos considerados. Además, en este estudio se analizó la ansiedad matemática, AP, AE y AG según la OCM y el género, y de acuerdo a la OCM, según género y país, pero no se encontraron efectos de interacción significativos en ninguno de los dos casos. En relación con ello, Pérez-Tyteca (2012) encontró una ansiedad matemática, AP y AE significativamente mayor en las mujeres que en los hombres en cada nivel de OCM, resultados que coincidieron con investigaciones como las de Fennema y Sherman (1976), Wigfield y Meece (1988) o Gil et ál. (2005). Pérez-Tyteca (2012) también encontró una AG muy similar entre hombres y mujeres en cada nivel de OCM.

Se encontró gran similitud entre la AM del estudiantado español y la AM del estudiantado costarricense, ya que las diferencias no fueron estadísticamente significativas, lo cual es un dato preocupante dado que en ambos países se presenta, entonces, ansiedad matemática en el estudiantado. Este resultado permite poder utilizar y aplicar en Costa Rica programas de disminución de AM que ya se han diseñado y aplicado en España, por ejemplo, los programas realizados por Caballero (2013), Iriarte y Benavides (2011) e Iriarte y Sarabia (2012), y no habría que empezar desde cero diseñando programas para reducir la ansiedad, o quizá, podríamos inspirarnos en ellos para crear programas propios que se ajusten más al perfil del estudiantado costarricense, ya que hemos visto, por ejemplo, que sí existen diferencias en la ansiedad matemática hacia los problemas y la ansiedad matemática hacia los exámenes entre ambos países. Al respecto, Gil-Flores y García-Gómez (2017) encontraron una correlación positiva entre el rendimiento del alumnado español y las variables que reflejan el desarrollo social y económico; estos autores se centran en analizar el papel de las políticas educativas regionales y de las instituciones escolares en la explicación de las diferencias de rendimiento de estudiantado español; así, recomendamos incluir como variables la influencia de las políticas educativas regionales y de las instituciones escolares sobre la AM en la población estudiantil costarricense, tomando en cuenta que en España ya han sido estudiadas estas variables. Por su parte, la similitud entre la AM del estudiantado español y la AM del estudiantado costarricense no siempre ocurre, dado que en una comparación similar realizada por Mohamed y Tarmizi (2010) entre estudiantes de Malasia y de Tanzania, concluyeron que había una mayor AM en estudiantes de Malasia que en Tanzania.

Entre las recomendaciones de cara a realizar trabajos futuros en esta misma línea tenemos las siguientes: (a) Analizar a profundidad el comportamiento en los subconstructos AP o AE, con el fin de determinar variables de cada país que puedan estar influyendo en ellos. (b) Profundizar en el hecho de que el género no presente un efecto significativo sobre el subconstructo AG. (c) Realizar más estudios con el fin de clarificar las causas del comportamiento diferenciado de la 
http://doi.org/10.15359/ree.24-2.15

ROR: https://ror.org/01t466c14 Universidad Nacional, Costa Rica http://www.una.ac.cr/educare

educare@una.cr

AM, AP, AE y AG respecto a la variable OCM en ambos países. (d) Corroborar el motivo por el cual no se encontraron efectos de interacción mutua de las variables género y OCM con respecto a la AM, ni con respecto a cada uno de los tres subconstructos considerados, ni en la AM, AP, AE y AG según la OCM y el género, ni de acuerdo a la OCM según género y país, y en otras investigaciones sí se dieron esas diferencias. Aunque posiblemente esta discrepancia puede ser causada por las diferencias entre titulaciones de ambos países, también podría estar relacionado con la manera de trabajar la resolución de problemas en ambos países o con el peso de las evaluaciones, sería conveniente corroborarlo. (e) Aplicar en Costa Rica programas de disminución de AM que ya se han diseñado y aplicado en España, por ejemplo, los programas realizados por Caballero (2013), Iriarte y Benavides (2011) e Iriarte y Sarabia (2012). (f) Indagar sobre los factores dados en cada país, los cuales podrían estar influyendo en la similitud del comportamiento de la AM, con el fin de detectar los que aumentan o disminuyen la AM sufrida por estudiantes $y$, con ello, sus consecuencias en el proceso de enseñanza aprendizaje de las matemáticas.

\section{Referencias}

Caballero, A. (2013). Diseño, aplicación y evaluación de un programa de intervención en control emocional y resolución de problemas matemáticos para maestros en formación inicial [Tesis doctoral]. Universidad de Extremadura, España. http://hdl.handle.net/10662/590

De la Torre, E., Mato, M. D. y Rodríguez, E. R. (2009). Ansiedade e rendemento en matemáticas. Eduga. Revista Galega do Ensino, 56, 73-77. https://dialnet.unirioja.es/servlet/ articulo?codigo $=3053016$

Delgado, I. C. (2015). Ansiedad matemática en los estudiantes del curso MAT-001 Matemática General de la Universidad Nacional durante el I ciclo del 2015, su comportamiento y las variables relacionadas a esta [Tesis de licenciatura]. Universidad Nacional, Costa Rica, Heredia.

Fennema, E. y Sherman, J. A. (1976). Fennema-Sherman mathematics attitudes scales: Instruments designed to measure attitudes toward the learning of mathematics by females and males. Journal for research in Mathematics Education, 7(5), 324-326. https:// doi.org/10.2307/748467

Gil, N., Blanco, L. J.y Guerrero, E. (2005). El dominio afectivo en el aprendizaje de las matemáticas. Una revisión de sus descriptores básicos. Unión. Revista Iberoamericana de Educación Matemática, 2, 15-32. https://dialnet.unirioja.es/servlet/articulo?codigo=2218956

Gil-Flores, J. y García-Gómez, S. (2017). Importancia de la actuación docente frente a la política educativa regional en la explicación del rendimiento en PISA. Revista de Educación, 378, 52-74. http://www.educacionyfp.gob.es/revista-de-educacion/dam/jcr:68887783-86fa4216-b71c-d57765aa3583/03garcia-pdf.pdf 
http://doi.org/10.15359/ree.24-2.15

ROR: https://ror.org/01t466c14 Universidad Nacional, Costa Rica

http://www.una.ac.cr/educare

educare@una.cr

Hannula, M. S. (2011). The structure and dynamics of affect in mathematical thinking and learning. En M. Pytlak, T. Rowland y E. Swoboda (Eds.), Proceedings of the Seventh Congress of the European Society for Research in Mathematics Education (pp. 34-60). University of Rzesów.

Hidalgo, S., Maroto, A., Ortega, T. y Palacios, A. (2013). Influencia del dominio afectivo en el aprendizaje de las matemáticas. En V. Mellado, L. J. Blanco, A. B. Borrachero y J. A. Cárdenas (Coords.), Las emociones en la enseñanza y el aprendizaje de las ciencias experimentales y las matemáticas (pp. 217-242). DEPROFE.

Ho, H.-Z., Senturk, D., Lam, A. G., Zimmer, J. M., Hong, S., Okamoto, Y., Chiu, S. Y., Nakazawa, Y., Collee, S. W, J. y Wang, C.-P. (2000). The affective and cognitive dimensions of math anxiety: A cross-national study. Journal for research in Mathematics Education, 31(3), 362379. https://doi.org/10.2307/749811

Iriarte, C. y Benavides, M. (2011). Evaluación del programa para superar la ansiedad hacia las matemáticas PAM. INFAD. International Journal of Developmental and Educational Psychology, 1(2), 65-73. https://dialnet.unirioja.es/servlet/articulo?codigo $=5988643$

Iriarte, C. y Sarabia, A. (2012). Programa para superar la ansiedad hacia las matemáticas: PAM: Aplicación práctica para intervenir en las dificultades de aprendizaje (2. ${ }^{a}$ ed). Ulzama.

Lee, J. (2009). Universals and specifics of math self-concept, math self-efficacy, and math anxiety across 41 PISA 2003 participating countries. Learning and Individual Differences, 19(3), 355365. https://doi.org/10.1016/j.lindif.2008.10.009

Marshall, G. (2000). Explaining mathematics anxiety in college students: A research project. The Mathematics Educator, 5(1-2), 108-116.

McLeod, D. B. (1989). Beliefs, attitudes, and emotions: New views of affect in mathematics education. En B. Douglas y V. M. Adams (Eds.), Affect and mathematical problem solving. A new perspective (pp. 245-258). Springer. https://doi.org/10.1007/978-1-4612-3614-6

Mohamed, S. H. y Tarmizi, R. A. (2010). Anxiety in mathematics learning among secondary school learners: A comparative study between Tanzania and Malaysia. Procedia - Social and Behavioral Sciences, 8, 498-504. https://doi.org/10.1016/j.sbspro.2010.12.068

Organización para la Cooperación y el Desarrollo Económico (OCDE). (2004). Informe PISA 2003: Aprender para el mundo del mañana. Santillana.

Palacios, A., Hidalgo, S., Maroto, A. y Ortega, T. (2013). Causas y consecuencias de la ansiedad matemática mediante un modelo de ecuaciones estructurales. Enseñanza de las Ciencias: Revista de Investigación y Experiencias Didácticas, 31(2), 93-111. https://www.raco.cat/ index.php/Ensenanza/article/view/285760 
Pérez-Tyteca, P. (2012). La ansiedad matemática como centro de un modelo causal predictivo de elección de carreras [Tesis doctoral]. Universidad de Granada, España. http://hdl.handle. net/10481/23293

Pérez-Tyteca, P., Castro, E., Rico, L. y Castro, E. (2011). Ansiedad matemática, género y ramas de conocimiento en alumnos universitarios. Enseñanza de las Ciencias, 29(2), 237-250. https:// doi.org/10.5565/rev/ec/v29n2.570

Pérez-Tyteca, P., Castro, E., Segovia, I., Castro, E., Fernández, F. y Cano, F. (2009). El papel de la ansiedad matemática en el paso de la educación secundaria a la educación universitaria. PNA, 4(1), 23-35. http://hdl.handle.net/11441/42231

Perina, K. (2002). The sum of all fears. Psychology Today Magazine, 35(6), 19-19.

Petriz, M. A., Barona, C., López, R. M. y Quiroz, J. (2010). Niveles de desempeño y actitudes hacia las matemáticas en estudiantes de la licenciatura en administración en una universidad estatal mexicana. Revista Mexicana de Investigación Educativa, 15(47), 1223-1249. http:// www.scielo.org.mx/scielo.php?script=sci arttext\&pid=S1405-66662010000400012

Richardson, F. C. y Suinn, R. M. (1972). The mathematics anxiety rating scale: Psychometric data. Journal of CounselingPsychology, 19(6), 551-554. https://doi.org/10.1037/h0033456

Rodríguez, P., Hidalgo, S. y Palacios, A. (2012). La ansiedad matemática en alumnos de grados en estadística. En A. Estepa, Á. Contreras, J. Deulofeu, M. C. Penalva, F. J. García y L. Ordóñez (Eds.), Investigación en Educación Matemática XVI (pp. 469-478). SEIEM.

Sánchez-Mendías, J. (2013). Actitudes hacia las matemáticas de los futuros maestros de educación primaria [Tesis doctoral]. Universidad de Granada, España. http://hdl.handle. net/10481/29827

Tobias, S. y Weissbrod, C. (1980). Anxiety and mathematics: An update. Harvard Educational Review, 50(1), 63-70. https://doi.org/10.17763/haer.50.1.xw483257j6035084

Valero, L. (1999). Evaluación de ansiedad ante exámenes: Datos de aplicación y fiabilidad de un cuestionario CAEX. Anales de Psicología, 15(2), 223-231. http://www.redalyc.org/articulo. oa?id=16715206

Wigfield, A., y Meece, J. L. (1988). Math anxiety in elementary and secondary school students. Journal of educational Psychology, 80(2), 210-216. https://doi.org/10.1037/0022$\underline{0663.80 .2 .210}$ 